\title{
Abnahme von Inzidenz und Prävalenz der Demenz, aber Zunahme der Betroffenen
}

Fragestellung: Wie kann die Entwicklung der Anzahl von Demenzfällen in den kommenden Jahrzehnten valide vorhergesagt werden?

Hintergrund: Die durch eine Demenz mit alltagsrelevanter Behinderung gelebte und durch vorzeitigen Tod verlorene Lebenszeit (DALY) stieg in Großbritannien zwischen 1990 und 2010 um $76 \%$ an. Bisherige Schätzungen zur zukünftigen Entwicklung der Anzahl Demenzkranker in einer Population gingen von einer über den vorausgesagten Zeitraum unverändert bleibenden Prävalenz aus. Entsprechend wurden bis zum Jahr 2050 weltweit mehr als 100 Millionen Menschen mit Demenz prognostiziert. Eine möglichst hohe Genauigkeit solcher epidemiologischer Vorhersagen ist aufgrund ihrer Implikationen für die weitere gesundheitspolitische Planung von großer Bedeutung.

Patienten und Methodik: In den Jahren 2002 bis 2013 wurde eine repräsentative Stichprobe mit 17.906 über Fünfzigjährigen alle zwei bis vier Jahre bezügAhmadi-Abhari S, GuzmanCastillo M, Bandosz P et al. Temporal trend in dementia incidence since 2002 and projections for prevalence in England and Wales to 2040: modelling study. BMJ 2017; 358: j2856 lich vaskulärer Risikofaktoren, kardiozerebraler Ereignisse und Lebensstil interviewt, kognitiv getestet und die Teilnehmer selbst oder Angehörige zu Beeinträchtigungen bei Alltagsaktivitäten befragt. Eine Demenz wurde dann angenommen, wenn eine kognitive Beeinträchtigung und Schwierigkeiten bei mindestens einer Alltagsaktivität vorlagen, oder wenn zwischenzeitlich eine Demenz von einem Arzt diagnostiziert worden war. Für die Berechnung zeitlicher Veränderungen der Demenzinzidenz im Beobachtungszeitraum wurde ein Modell verwendet, das Veränderungen der Mortalitätsraten kardiovaskulärer Erkrankungen einkalkuliert und bezüglich einer Verzerrung durch Dropouts korrigiert. Für die Vorhersage der Demenzprävalenzen bis zum Jahr 2040 wurde ein probabilistisches, zeitlich diskretes Markov-Modell (IMPACT-BAM) angewandt, das jährliche Veränderungen von Wahrscheinlichkeiten mehrerer Parameter berücksichtigt.

Ergebnisse: Die Demenzinzidenz nahm jährlich um 2,7\% in den Jahren 2002 bis 2013 ab. Trotz der in allen Altersgruppen abnehmenden Inzidenz- und Prävalenzraten wurde für England und Wales eine Zunahme an Demenzkranken von 872.000, 1.092.000 und 1.205.000 für die Jahre 2020, 2030 und 2040 vorausberechnet. Daraus ergibt sich ein Anstieg der Anzahl von Demenzkranken zwischen 2016 und 2040 um $57 \%$. Bei Annahme einer konstanten Inzidenz würden im Jahr 2040 mit 1,9 Millionen deutlich mehr Demenzkranke erwartet.

Schlussfolgerungen: Die Inzidenz und Prävalenz von Demenzen nimmt in allen Altersgruppen ab. Trotzdem kommt es aufgrund der höheren Lebenserwartung zu einem deutlichen Anstieg der absoluten Häufigkeit von Demenzfällen.

\section{- Kommentar von Patrick Jung, Mainz/Frankfurt am Main}

\section{Gesunder Lebensstil wirkt präventiv}

Diese methodisch hochwertige Modellstudie bestätigt die Ergebnisse einer abnehmenden Inzidenz für Demenzen anderer kürzlich veröffentlichter Studien aus weiteren Industrienationen (Italien, Spanien, Niederlande, USA). Sie stützt die Annahme, dass das Vorhandensein vaskulärer Risikofaktoren wie arterielle Hypertonie, Diabetes mellitus, Rauchen, Hyperlipidämie, geringe körperliche Aktivität und Übergewichtigkeit das Risiko zur Entwicklung einer Demenz erhöht. Das therapeutisch bislang wenig ertragreiche Themengebiet der Demenzerkrankungen kann demnach eine positive Nachricht verzeichnen: Ein gesunder Lebensstil wirkt präventiv! Die Studie beantwortet allerdings nicht, ob präventive Maßnahmen eine Demenzerkrankung verhindern oder lediglich hinauszögern können. Die durch einen gesunden Lebensstil, verbesserte Hygiene und medizinische Versorgung erzielte höhere Lebenserwartung erhöht wiederum die Wahrscheinlichkeit, an einer Demenz zu erkranken, da das Alter den wichtigsten Risikofaktor der Demenz darstellt. Durch die stark zunehmende Anzahl älterer Men- schen in den kommenden Jahrzehnten erklärt sich daher die deutlich ansteigende absolute Häufigkeit der Demenzen trotz sinkender Inzidenz- und Prävalenzraten, sie bleibt jedoch deutlich hinter prognostizierten Zahlen bei angenommener gleichbleibender Inzidenz und Prävalenz zurück. Zeitlich dynamische Modellstudien wie diese sind daher von großer Bedeutung für die weitere gesundheitspolitische Planung.

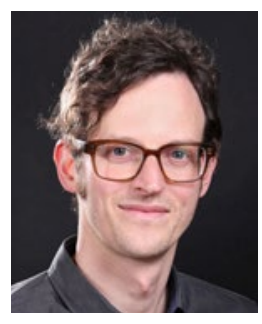

PD Dr. med. Patrick Jung, Mainz/

Frankfurt am Main

Universitätsmedizin Mainz, Klinik für Psychiatrie und Psychotherapie, Neuropsychiatrisches Zentrum Frankfurt-Sachsenhausen E-Mail: patrick.jung@unimedizin-mainz.de 